\title{
Sistema Supervisório e Controle Multivariável aplicando Controlador Fuzzy Ponderado-PID em um Processo de Fermentação Alcoólica
}

\section{Supervisory System and Multivariable Control Applying Weighted Fuzzy-PID Logic in an Alcoholic Fermentation Process}

\author{
Márcio Mendonça ${ }^{1}$; Elton Carlos Correa²; Ivan Rossato Chrun³; Orion Buss ${ }^{4}$
}

\begin{abstract}
Resumo
Neste trabalho, é analisado, o controle de um sistema multivariável, um processo de fermentação alcoólica com fase não mínima. O controle é feito com controladores clássicos, Proporcional, Integrativo e Derivativo (PID) associado a um sistema supervisório baseado em Sistemas Fuzzy. O sistema Fuzzy, a priori, será um passador de set-points aos controladores PID, porém também agrega funções de proteção, como por exemplo, caso a biomassa esteja com valor zero ou muito próximo; O controlador Fuzzy altera a campanha para evitar ou amenizar o problema de paralisar a reação química na cuba. Duas arquiteturas de controle baseadas em sistemas de controle Fuzzy serão apresentadas, e comparadas em desempenho com controle clássico em campanhas diferentes. Um breve resumo sobre teoria Fuzzy e alguns trabalhos correlatos também serão apresentados. E, finalmente resultados de simulações, conclusões e futuros trabalhos encerram o artigo.
\end{abstract}

Palavras-chave: Sistemas Supervisórios. Controle de um Sistema Multivariável. Processo de Fermentação Alcoólica. Controle Clássico. Controle Fuzzy-PID.

\begin{abstract}
In this work, it is analyzed a multivariate system control of an alcoholic fermentation process with no minimum phase. The control is made with PID classic controllers associated with a supervisory system based on Fuzzy Systems. The Fuzzy system, a priori, send set-points to PID controllers, but also adds protection functions, such as if the biomass valued is at zero or very close. The Fuzzy controller changes the campaign to prevent or mitigate the paralyzation of the process. Three control architectures based on Fuzzy Control Systems are presented and compared in performance with classic control in different campaigns. The third architecture, in particular, adds an adaptive function. A brief summary of Fuzzy theory and correlated works will be presented. And, finally simulations results, conclusions and future works end the article.
\end{abstract}

Keywords: Supervisory Systems. Multivariate Control System. Alcoholic Fermentation Process. Classic Control. Fuzzy-PID Control.

\footnotetext{
${ }^{1}$ Docente no departamento de Engenharia Elétrica, (DAELE), UTFPR-CP, Programa de Mestrado (COEME) Universidade Tecnológica Federal do Paraná, Campus Cornélio-Procópio; mendonca@utfpr.edu.br

${ }^{2}$ Aluno de graduação do Departamento de Engenharia Elétrica, Universidade Tecnológica Federal do Paraná, Campus Cornélio Procópio; eltoncorrea.utfpr@gmail.com

${ }^{3}$ Aluno de mestrado na Tecnológica Federal do Paraná, Campus Curitiba (CPGEI)

${ }^{4}$ Aluno de Graduação do Departamento de Engenharia Elétrica, Universidade Tecnológica Federal do Paraná, Campus Cornélio Procópio; orion.buss@gmail.com
} 


\section{Introdução}

A lógica Fuzzy é baseada na teoria dos conjuntos Fuzzy. Ela difere dos sistemas lógicos tradicionais em suas características e seus detalhes, a rigor a lógica clássica é uma generalização devida sua capacidade de modelar somente zeros e uns, estados limites das funções de pertinência. De outro modo, nessa lógica, o raciocínio exato corresponde a um caso limite do raciocínio aproximado, sendo interpretado como um processo de composição de relações nebulosas (GOMIDE, PEDRIKS, 2006).

A motivação da aplicação dessa técnica está na capacidade de seres humanos de lidar com processos bastante complexos, baseados em informações imprecisas e/ou aproximadas. A estratégia adotada é também de natureza imprecisa e geralmente possível de ser expressa em termos linguísticos. (PASSINO; YOURKOVICH, 1997).

Este trabalho aborda o desenvolvimento de um sistema supervisório com algumas funções de proteção para um processo de fermentação alcoólica, utilizando Sistemas Fuzzy. A arquitetura clássica de controle supervisório pode ser vista na literatura, como por exemplo, o trabalho de Passino e Yourkovich (1997).

No processo em estudo, o controle é multivariável, no qual, controladores PID (Proporcional Integral e Derivativo) determinam a abertura e fechamento das válvulas Fin (Válvula de Entrada) e Fout (Válvula de Saída), a primeira é responsável pelo fluxo de substrato inserido no tanque e a segunda pelo fluxo de produto fermentado retirado da cuba. Vale ressaltar que: a modelagem do sistema e os controladores clássicos são similares do trabalho de Mendonça (2011).

Essa pesquisa desenvolve um sistema supervisório para o processo de fermentação alcoólica, com duas arquiteturas de controle, diferentes quanto à utilização da lógica Fuzzy simultaneamente com controle PID. Estas arquiteturas estão descritas a seguir: na primeira arquitetura um controlador Fuzzy passa os setpoints para os controladores PID; na segunda um controlador Fuzzy passa os set-points para os controladores PID quando o sistema está em condições de alarmes, e um segundo controlador Fuzzy assume a posição com o sistema operando em condições normais.

Segundo Shaw e Simões (2004) variáveis isoladas do processo são controladas por PID, enquanto o controle supervisório é feito por operadores humanos. Existem sistemas supervisórios comerciais, como, por exemplo, Elipse que implementa uma lógica clássica própria. A lógica Fuzzy pode fornecer uma solução eficiente para esse problema, pelos quais controladores supervisórios multivariáveis Fuzzy são projetados com base na experiência (conhecimento heurístico) dos operadores e/ ou especialistas, e não se utilizar modelos matemáticos complexos.

A metodologia empregada no desenvolvimento do trabalho é de simular o processo de fermentação alcoólica, através das equações diferenciais que descrevem o comportamento dinâmico do processo. As simulações são feitas no software MATLAB, utilizando o método de Runge-Kutta de $4^{\mathrm{a}}$ ordem para a resolução das equações.

Este trabalho está dividido da seguinte forma: na segunda seção é feita uma breve revisão bibliográfica de trabalhos correlatos que utilizam Sistemas Fuzzy em controle e supervisão de processos; a terceira seção apresenta uma breve introdução de sistemas Fuzzy e suas principais vantagens e desvantagens; a quarta seção descreve e modela o sistema de fermentação alcoólico; a quinta seção apresenta o desenvolvimento do sistema supervisório para o processo de fermentação alcoólica, e, em seguida compara os resultados obtidos com o controle clássico PID; a sexta seção apresenta resultados e comportamentos dinâmicos das arquiteturas de 
controle propostas.; e, finalmente a última seção faz à conclusão do trabalho, e sugestões para trabalhos futuros.

\section{Trabalhos correlatos com sistemas Fuzzy}

O universo de aplicações em diferentes áreas da Lógica Fuzzy é bastante extenso (PASSINO; YOURKOVICH, 1997). Entretanto, alguns trabalhos correlatos serão apresentados como forma de motivação dessa pesquisa.

O trabalho de Yang et al. (2010) utiliza um controlador Fuzzy Ponderado em um moinho de esfera, processo industrial que se utiliza de conceitos de química, metalurgia e energia elétrica. Esse processo é um complexo mecanisno mecânico; com ponto de controle crítico quando se relaciona capacidade de produção e eficiência energética. Nesse contexto, um controlador com regras ajustáveis obteve resultados da ordem de $25 \%$ melhores do que o PID clássico, segundo os autores.

Fernandes (2005) sugere um modelo de supervisão utilizando lógica Fuzzy para um sistema de geração de energia híbrido, este modelo é composto por um grupo gerador diesel, um gerador eólico e um banco de baterias. O objetivo é fazer o gerador diesel trabalhar o menos possível para economizar combustível; o banco de baterias atua quando o gerador eólico não consegue suprir a demanda de energia por um curto intervalo de tempo, onde não é viável utilizar o gerador diesel, conseguindo uma eficiência maior no uso do gerador eólico.

Yamakawa (2007) propõe um sistema de controle Fuzzy para banco de capacitores automático, aplicado nos alimentadores de distribuição de energia elétrica com o intuito de minimizar o número de chaveamento do banco de capacitores sem alterar os níveis de tensão do alimentador, otimizando o desempenho do equipamento, resultando em uma maior correção dos reativos.
O artigo do Yesil et al. (2013) apresenta um FCM (Fuzzy Cognitive Maps) para a sintonia dos parâmetros de controladores PI aplicado a um sistema não linear. Os controladores PI, para sistemas deste tipo, não conseguem resultados satisfatórios, por causa de diferentes propriedades estáticas e dinâmicas, porém esse trabalho mostrou uma solução eficiente para esse problema com o ajuste online dos parâmetros do controlador PI para cada ponto de operação do sistema, através de um FCM.

O trabalho de Arriaga-De-Valle e Dieck-Assad (2006) discute e compara o controle clássico PID aplicado a um sistema de caldeira para geração de vapor, com um sistema Fuzzy supervisório gerando set-points para o controle clássico. O sistema supervisório proporcionou uma economia de combustível de 2,5 a 6,5\%, dependendo da carga de vapor.

No trabalho Mendonça (2011) apresenta uma Rede Cognitiva Dinâmica, do inglês Dynamic Cognitive Networks, uma evolução dos Fuzzy Cognitive Maps, para controle e sistema supervisório do mesmo processo, fermentador alcoólico, similar ao utilizado nessa pesquisa.

O trabalho Wang et al. (2012) utiliza um controlador Fuzzy-PID para elevadores. Com o objetivo de reduzir o grande consumo de energia, este trabalho apresenta um novo sistema de controle que pode transformar a energia do motor para trabalhar no estado gerador à rede elétrica. Esse sistema de controle é necessário porque o controle PI convencional não pode funcionar de forma eficiente no controle do variável tempo e de objetos não lineares.

\section{Vantagens e desvantagens da lógica Fuzzy}

A lógica Fuzzy foi proposta pelo professor de ciências da computação Lotfi A. Zadeh em meados da década de 60 (ZADEH, 1992). O modelo de controle Fuzzy Mamdani, utilizado 
nesse trabalho, foi criado por Ebrahim Mamdani em 1975, para controlar a caldeira de uma máquina a vapor; através de um conjunto de regras baseada em variáveis linguísticas, regras que foram construídas por meio do conhecimento de operadores (PASSINO; YOURKOVIC, 1997).

A idéia básica em controle fuzzy é modelar as ações a partir de conhecimento especialista, ao invés de, necessariamente, modelar o processo em si. As motivações para esta nova abordagem estão nos casos nos quais o conhecimento de um ou mais especialistas de controle são possíveis de serem obtidos. Seja por meio de operadores ou de projetistas ou até mesmo de ambos. Enquanto que os modelos matemáticos envolvidos são em geral custosos, ou complicados para serem desenvolvidos (GOMIDE; GUDWIN, 1994).

De um modo geral, um sistema de controle Fuzzy clássico, como utilizado nesse trabalho, pode ser desenvolvido com o conhecimento de um especialista do processo por meio de um conjunto de regras, nas quais as condições são dadas a partir de um conjunto de termos linguísticos associados às variáveis de entrada e saída do processo. (GOMIDE; PEDRYCZ, 2006).

Não é escopo desse trabalho apresentar os mecanismos de funcionamento da lógica Fuzzy e/ou as operações matemáticas utilizadas para fuzzificação e defuzzicação dos dados. Limita-se a somente a uma breve introdução e algumas das vantagens e desvantagens dessa técnica.

\section{Vantagens de se utilizar lógica Fuzzy:}

- Abstração e modelagem pode ser feita por meio de conhecimentos expressos em termos linguísticos; a linguagem natural, que é usado por pessoas comuns em uma base diária.

- Uma abordagem mais intuitiva, sem a complexidade de longo alcance e flexível;
- Tolerante com dados imprecisos;

- A lógica Fuzzy pode modelar funções não lineares de complexidade arbitrária.

- Pode-se desenvolver um sistema Fuzzy para combinar com qualquer conjunto de dados de entrada e saída. Através de técnicas adaptativas (Alisson, 2014), como por exemplo, Adaptive Neuro-Fuzzy Inference Systems (ANFIS);

- Pode-se desenvolver controladores, por meio do conhecimento e experiência de um ou mais especialistas e operadores;

- Em contraste direto com Redes Neurais Artificiais, que utilizam dados históricos para treinamento, a lógica Fuzzy permite contar com o conhecimento, e a experiência de pessoas que já entendem o sistema; entretanto dados podem ser usados para refinamento do modelo.

- A lógica Fuzzy pode ser usada em conjunto com técnicas de controle convencionais (caso dessa pesquisa);

Porém, se uma solução mais simples já existe, esta é recomendada. Entretanto é uma ferramenta sugerida para lidar com rapidez e eficiência problemas com imprecisão e não linearidade (KASABOV, 1998). Outra desvantagem, não só da Lógica Fuzzy, mas também de sistemas computacionais inteligentes, de um modo geral, não se garante que o resultado final seja ótimo, porém é possível se obter soluções em situações onde não se conhece ou somente parcialmente os modelos matemáticos de um sistema, por exemplo (MENDONÇA, 2011).

\section{Processo Fermentação Alcoólico}

De um modo geral, existem duas válvulas que controlam, respectivamente, o fluxo de substrato inserido no tanque e o fluxo de produto (fermentado) retirado. Estas válvulas são 
controladas por dois controladores independentes do tipo Proporcional - Integral - Derivativo (PID).

O processo de fermentação alcoólica utilizado neste artigo, foi inicialmente estudado por Maher (1995). Pode-se observar na figura 1, que o processo possui quatro variáveis de estado: as concentrações de substrato $(\mathbf{S})$, de biomassa $(\mathbf{C})$, de produto $(\mathbf{P})$ e o volume $(\mathbf{V})$ do tanque onde ocorre a fermentação. As concentrações são dadas em gramas/litro (g/l), e o volume em litros (1). A figura 1 também apresenta as válvulas Fin e Fout onde, a primeira é responsável pelo fluxo de substrato inserido no tanque e a segunda pelo fluxo de produto fermentado retirado da cuba.

Figura 1 - Processo de Fermentação Alcoólica

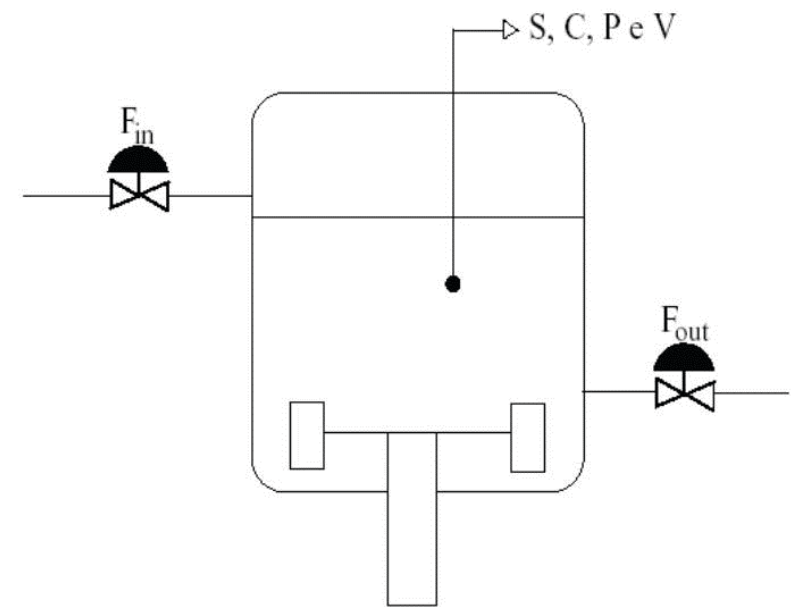

Fonte: Adaptado de Maher (1995).

Este processo apresenta interação entre suas variáveis, o que resulta em não linearidade. A análise da curva de resposta ao degrau, na figura 2, mostra um comportamento de fase não mínima e tempo de acomodação grande (FABRO, 2003; MENDONÇA, 2011). Além disso, por medida de segurança, o volume do reservatório não deve exceder 3,5 1 nem ficar abaixo de 1,5 1, e para garantir a fermentação, a concentração de biomassa não deve superar $8 \mathrm{~g} / 1$, do mesmo modo a concentração de substrato não pode ficar abaixo de $0,5 \mathrm{~g} / \mathrm{l}$, porque com valores muito próximos a zero da biomassa a reação química se extingue.
Figura 2 - Comportamento Dinâmico das Variáveis de Estado do Processo de Fermentação Alcoólica

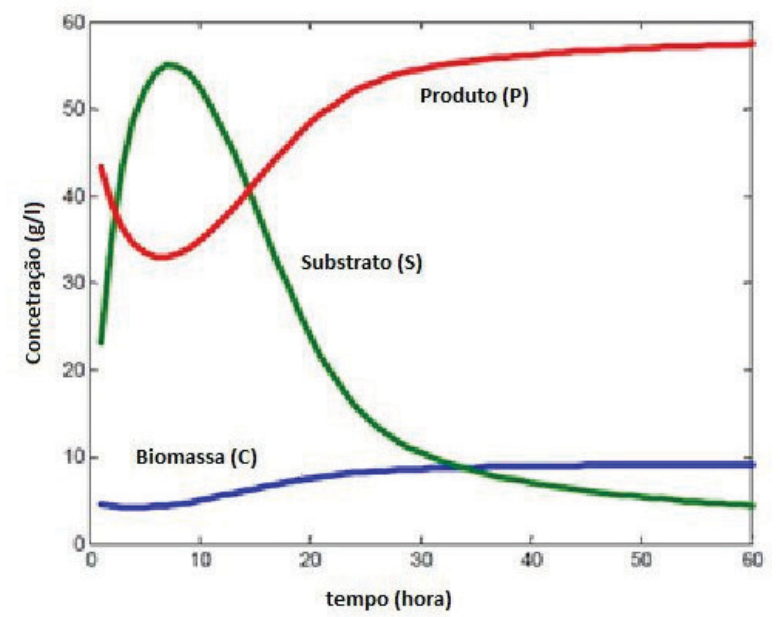

Fonte: o próprio autor

As equações diferenciais que descrevem o comportamento dinâmico do processo são dadas de (1) a (4).

$\frac{d S}{d t}=-\frac{1}{Y_{c} / s} \mu C+\frac{\text { Fin }}{V} S a-\frac{\text { Fout }}{V} S$

$\frac{d C}{d t}=\mu_{C}-\frac{\text { Fout }}{V} S$

$\frac{d P}{d t}=-\frac{Y_{p} / s}{Y_{c} / s} \mu C-\frac{\text { Fout }}{V} P$

$\frac{d V}{d t}=$ Fin - Fout

Onde:

$S a \rightarrow$ concentração de substrato da alimentação;

$\mathrm{Yc} / \mathrm{s} \rightarrow$ constante de conversão da biomassa;

$\mathrm{Yp} / \mathrm{s} \rightarrow$ constante de conversão do produto;

$\mu \rightarrow$ função de crescimento da biomassa, dada pela equação (5).

$\mu=\mu_{0} \frac{S}{K_{s}+S_{s}}\left(1-\frac{P}{P_{m}}\right)$ 
Onde:

$\mu_{-} 0 \rightarrow$ máxima taxa de crescimento;

$\mathrm{Ks} \rightarrow$ constante de Michaelis-Menton;

$\mathrm{Pm} \rightarrow$ coeficiente de inibição do produto durante a reação.

\section{Sistema de controle e supervisório Fuzzy}

Como já citado, essa pesquisa desenvolve duas formas de aplicação de controle Fuzzy juntamente com controladores PID, para simulação do processo, análise e comparação de resultados é utilizado duas campanhas de fermentação distintas, de tal modo que as duas arquiteturas de controle são testadas nessas duas campanhas, mostradas nas tabelas 1 e 2 .

Tabela 1 - Campanha 1

\begin{tabular}{cc}
\hline \multicolumn{2}{c}{ CAMPANHA 01 } \\
\hline $\begin{array}{c}\text { Tempo até } \\
\text { (horas) }\end{array}$ & $\begin{array}{c}\text { Concentração de produto desejado } \\
(\mathrm{g} / \mathrm{l})\end{array}$ \\
\hline 60 & 40 \\
120 & 45 \\
150 & 35 \\
200 & 40 \\
350 & 45 \\
\hline
\end{tabular}

Fonte: o próprio autor

Tabela 2 - Campanha 2

\begin{tabular}{cc}
\hline \multicolumn{2}{c}{ CAMPANHA 02 } \\
\hline $\begin{array}{c}\text { Tempo até } \\
\text { (horas) }\end{array}$ & $\begin{array}{c}\text { Concentração de produto desejado } \\
(\mathrm{g} / \mathrm{l})\end{array}$ \\
\hline 60 & 40 \\
120 & 15 \\
180 & 35 \\
240 & 45 \\
350 & 20 \\
\hline
\end{tabular}

Fonte: o próprio autor

Nas duas campanhas citadas, os mesmos startap mostrados utilizam os valores das constantes utilizadas para a evolução das equações diferencias, e podem ser vistos na tabela 3 , já os valores iniciais das variáveis $\mathrm{S}, \mathrm{C}, \mathrm{P}$ e V são mostrados na tabela 4.

A Primeira Arquitetura de Controle: Nesta arquitetura de controle supervisório apenas um controlador Fuzzy passa os set-points aos controladores PID, com o intuito de melhorar o processo de fermentação alcoólica, e alcançar funções de proteção para o S, C e V. No diagrama de bloco da figura 3 podemos observar o princípio do funcionamento desta arquitetura de controle supervisório, onde o processo é controlado pelos controladores PID e o sistema Fuzzy atua em nível supervisório passando os set-points aos controladores clássicos.

Figura 4 apresenta a estrutura do primeiro controlador Fuzzy, nota-se que este controlador possui cinco entradas e duas saídas. Para a entrada foi levado em consideração à variação do ponto de operação desejado (variação da concentração do produto fermentado, ou set-point do processo), o erro do ponto de operação além das concentrações de biomassa e substrato, e por último o volume do tanque. Enquanto as saídas - Fator Fint e Fator Fout - são fatores de multiplicação que controlam os valores dos set-points efetivamente aplicados aos controladores PID, que por sua vez controlam a abertura e fechamento das válvulas Fint e Fout.

Tabela 3 - Valores iniciais das constantes

\begin{tabular}{cc}
\hline \multicolumn{2}{c}{ CONSTANTES } \\
\hline $\mathrm{S} a$ & $100 \mathrm{~g} / \mathrm{l}$ \\
$\mathrm{Pm}$ & 100 \\
$\mathrm{Ks}$ & 10 \\
$\mu_{0}$ & 0,31 \\
$\mathrm{Yp} / \mathrm{s}$ & 0,07 \\
$\mathrm{Yc} / \mathrm{s}$ & 0,44
\end{tabular}

Fonte: o próprio autor

Tabela 4 - Variáveis do processo

\begin{tabular}{cc}
\hline \multicolumn{2}{c}{ VARIÁVEIS DO PROCESSO } \\
\hline S (Substrato) & $4,5 \mathrm{~g} / 1$ \\
C (Biomassa) & $5 \mathrm{~g} / 1$ \\
P (Produto) & $50 \mathrm{~g} / 1$ \\
V (Volume) & 21 \\
\hline
\end{tabular}

Fonte: o próprio autor 
Figura 3 - Arquitetura I de Controle Supervisório com um Sistema Fuzzy

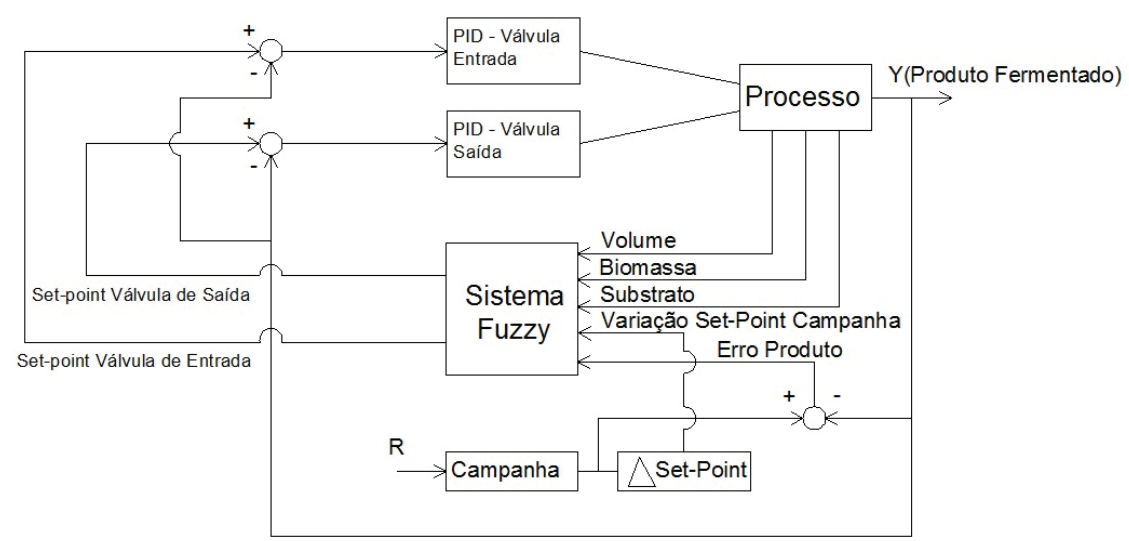

Fonte: o próprio autor

Figura 4 - Estrutura do controlador I (5x2)

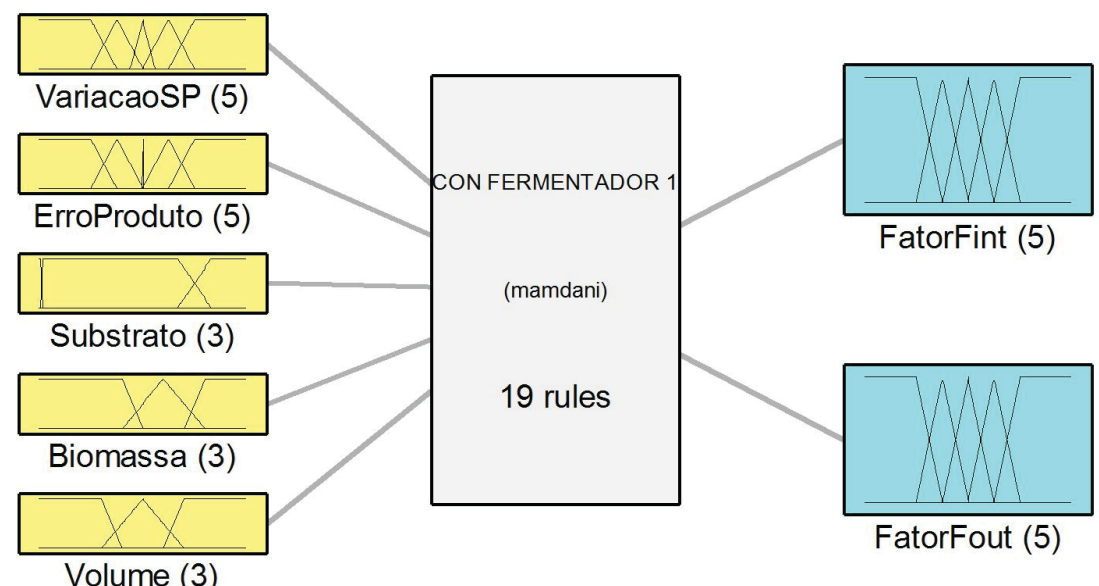

Fonte: o próprio autor

A tabela 5 descreve a heurística de desenvolvimento utilizada de forma resumida. A tabela 6 mostra algumas restrições de operação do processo de acordo com os trabalhos de (MENDONÇA, 2011), (FABRO, 2003).

A figura 5 mostra um exemplo de funções de pertinências utilizadas na elaboração dos controladores Fuzzy, nota-se que as funções das extremidades POUCO e MUITO são do tipo trapezoidal já a função do meio SUFICIENTE é do tipo triangular, vale ressaltar que para a construção das demais funções, das outras variáveis foi utilizado este mesmo modelo exposto como exemplo. Uma vez que as funções do tipo trapezoidal e triangular são aproximações por retas do ponto de referência desejado, quando não possui total domínio do problema. 
Tabela 5 - Descrição da observação processo RELAÇÕES DE CONTROLE DO PROCESSO

\begin{tabular}{l} 
AELAÇÕES DE CONTROLE DO PROCESSO \\
\hline A concentração de produto no reator, variável controlada \\
do processo, é afetada por variações no set-point do PID que \\
controla a válvula de entrada. \\
A concentração de produto no reator, variável controlada \\
do processo, é afetada por variações no set-point do PID que \\
controla a válvula de saída. \\
O valor do set-point desejado no processo, que caracteriza o \\
ponto de operação da reação, determina o valor de set-point \\
efetivamente aplicado ao PID, que controla a válvula de entrada. \\
O valor do set-point desejado no processo, que caracteriza o \\
ponto de operação da reação, determina o valor de set-point \\
efetivamente aplicado ao PID, que controla a válvula de saída. \\
A variação de set-point desejado (mudança de ponto de \\
operação) afeta o valor do set-point aplicado à válvula de \\
entrada. \\
A variação de set-point desejado (mudança de ponto de \\
operaçâo) afeta o valor do set-point aplicado à válvula de saída. \\
O volume do tanque é afetado pelo valor do set-point da válvula \\
Fin e Fout. \\
As concentrações de substrato e biomassa são afetadas \\
diretamente pela abertura e fechamento da válvula de entrada, \\
uma vez que essa válvula é responsável pelo fluxo de biomassa e \\
substrato que entra na cuba. \\
As concentrações de substrato e biomassa não são afetadas pela \\
abertura e fechamento da válvula de saída, sabendo que a válvula \\
de saída controla apenas o fluxo de produto fermentado que sai \\
do tanque.
\end{tabular}

Fonte: o próprio autor

Tabela 6: Restrições processo

\begin{tabular}{ll}
\hline RESTRIÇÕES DE OPERAÇÃO DO PROCESSO \\
\hline A quantidade de biomassa não deve superar uma \\
concentração de $8 \mathrm{~g} / \mathrm{l}$. \\
O volume do reator por questões de segurança não pode \\
exceder 3,5 litros nem ficar abaixo de 1,5 litros. \\
A quantidade de substrato não pode ficar abaixo de uma \\
concentração mínima de $0,5 \mathrm{~g} / \mathrm{l}$. \\
Os valores de set-points para concentração de produto \\
correspondem a uma lista de valores de concentração \\
discreta no intervalo $[10,50]$ g/l descrevendo os pontos de \\
operação.
\end{tabular}

Fonte: o próprio autor

Figura 5 - Exemplo de funções de pertinência (funções de pertinência para o controle do volume)

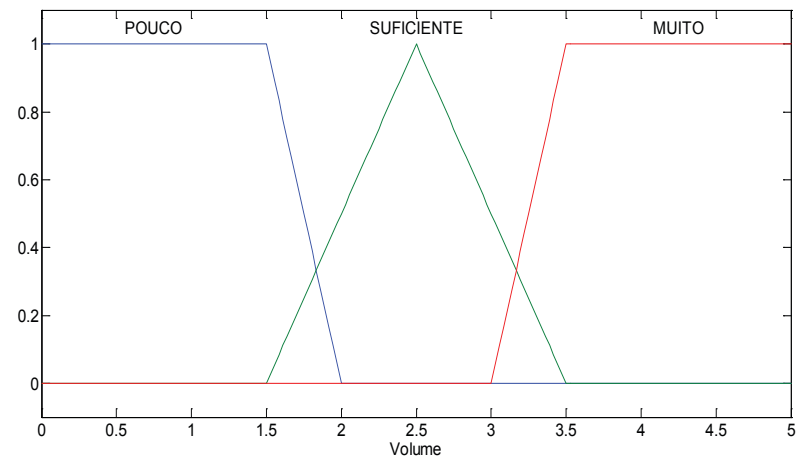

Fonte: o próprio autor
O quadro 1 apresenta, como exemplo, oito das dezenove regras do controlador Fuzzy da primeira arquitetura, as cinco primeiras atuam nas alterações dos pontos de operação no decorrer na campanha levando em consideração a variação do set-point do processo, as três ultimas são responsáveis pelo controle do volume do tanque.

Quadro 1: Oito das dezenove regras do controlador I

1. SE (Variação SP é MUITO negativa) ENTÃO (Fator Fint AUMENTA bastante) (Fator Fout AUMENTA bastante)

2. SE (Variação SP é negativa) ENTÃO (Fator Fint AUMENTA) (Fator Fout AUMENTA)

3. SE (Variação SP é mínima) ENTÃO (Fator Fint NÃO altera) (Fator Fout NÃO altera)

4. SE (Variação SP é positiva) ENTÃO (Fator Fint DIMINUI) (Fator Fout DIMINUI)

5. SE (Variação SP é MUITO positiva) ENTÃO (Fator Fint DIMINUI bastante) (Fator Fout DIMINUI bastante)

17. SE (Volume é POUCO) ENTÃO (Fator Fint AUMENTA BASTANTE) (Fator Fout DIMINUI BASTANTE)

18. SE (Volume é SUFICIENTE) ENTÃO (Fator Fint NÃO ALTERA) (Fator Fout NÃO ALTERA)

19. SE (Volume é MUITO) ENTÃO (Fator Fint DIMINUI BASTANTE) (Fator Fout AUMENTA BASTANTE)

Observa-se que, esses controladores são sistemas Fuzzy ponderados (YANG, et al., 2010), devido a necessidade de melhor sintonia do controlador para diferentes campanhas, no caso desse trabalho. Desse modo suas regras serão ponderadas por diferentes setups de conjunto de pesos para cada campanha diferente.

Figura 6 mostra a ponderação de cada regra do controlador Fuzzy da primeira arquitetura, ajustadas para a campanha 1, enquanto na figura 7 as regras estão ajustadas para a campanha 2. Para este controlador que trabalha tanto em condições normais de operação quanto em condições de alarme, esse ajuste de ponderação de acordo com a campanha foi essencial para o sucesso do controlador. 
Figura 6 - Ponderação das Regras do Controlador da Primeira Arquitetura de Controle, Ajustada para a Campanha

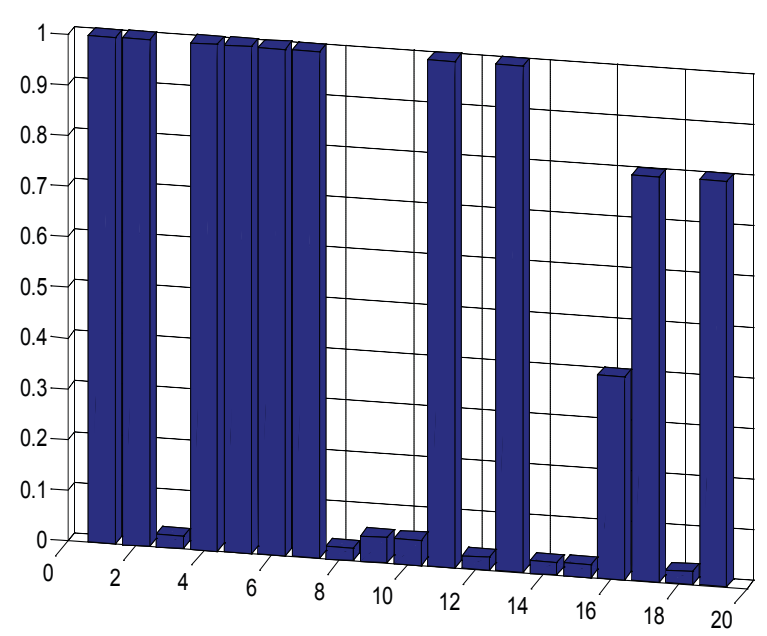

Fonte: o próprio autor

As superfícies fuzzy do controlador I (figuras 8 e 9) são mostradas na sequência. É possível observar a não linearidade e ações de controle diferentes nas duas válvulas.

Figura 7 - Ponderação das Regras do Controlador da Primeira Arquitetura de Controle, Ajustada para a Campanha

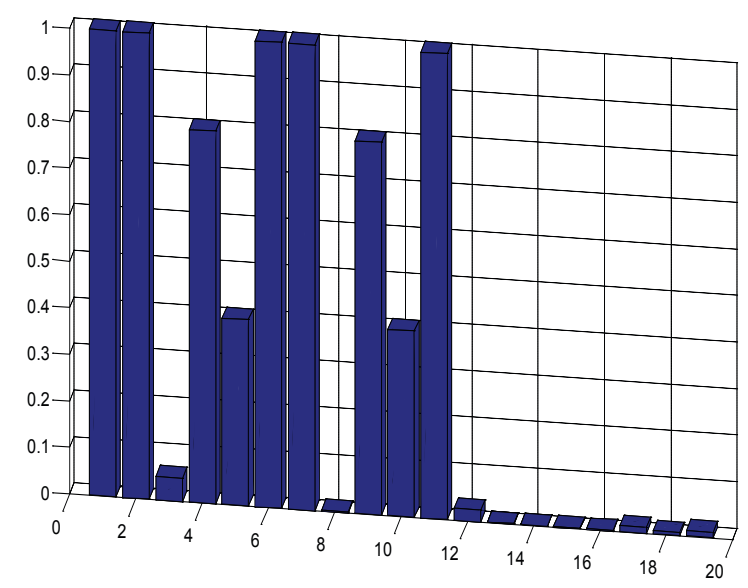

Fonte: o próprio autor
Figura 8 - Exemplo de Superfície, controlador I (Fin)

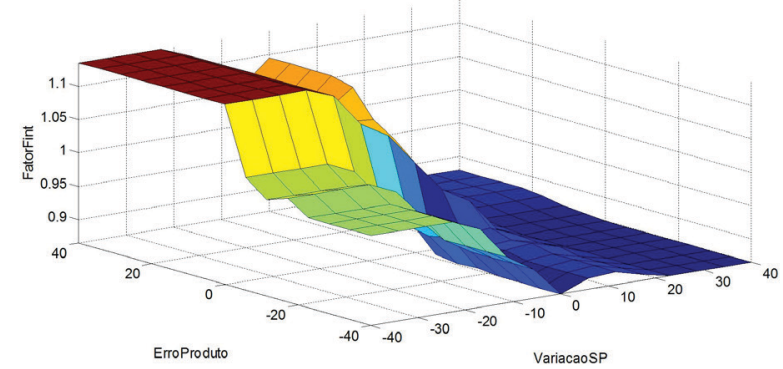

Fonte: o próprio autor

Figura 9 - Exemplo de Superfície, controlador I (Fout)

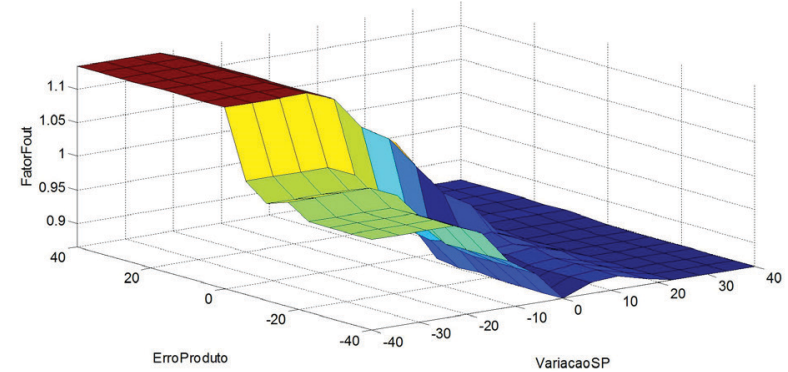

Fonte: o próprio autor

A segunda arquitetura de controle supervisório segue o mesmo princípio da primeira, no entanto utiliza-se dois sistemas Fuzzy, como mostrado na figura 10 .

A ideia desta arquitetura é dividir o sistema Fuzzy em dois níveis de atuação, o primeiro nível é representado pelo bloco de controle Fuzzy da direita. Este controlador é exatamente o mesmo da primeira arquitetura de controle (figura 3) com as devidas configurações para a campanha 01 e 02 .

O controlador Fuzzy do primeiro nível atua quando o processo de fermentação alcoólica se encontra em situações de alarmes, relacionados às restrições de operação do processo apresentados na tabela 6. 
Figura 10 - Arquitetura II (dois controladores)

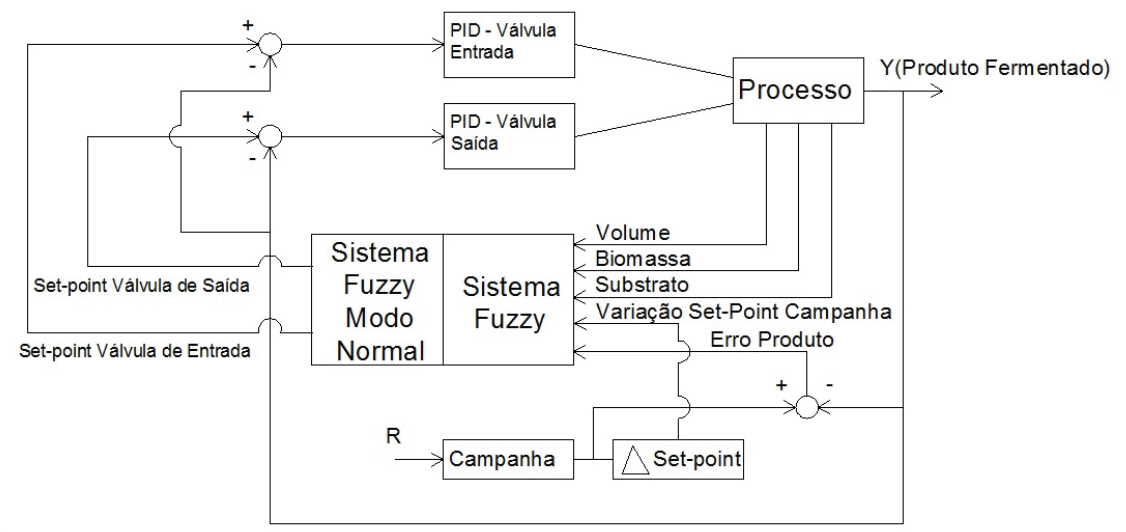

Fonte: o próprio autor

O segundo nível de atuação do sistema Fuzzy é simbolizado pelo bloco de controle da esquerda, suas características de projeto serão expostas de maneira subsequente.

O segundo nível de supervisão entra em ação quando o processo não apresenta situações de alarmes nas concentrações de substrato, biomassa e no volume do tanque de fermentação.

A transição do primeiro nível de atuação do sistema Fuzzy para o segundo nível é feita através da lógica clássica se/então.
O controlador Fuzzy do segundo nível é exposto na figura 11, note-se que o sistema é do tipo $2 \times 2$ e mantêm as duas primeiras entradas e as mesmas saídas, do controlador da primeira arquitetura de controle. Estas variáveis também continuam com as mesmas funções de pertinência. Assim, o que diferencia este controlador são as regras e suas ponderações, além de desconsiderar o volume do tanque e as concentrações de substrato e biomassa.

Figura 11 - Estrutura do controlador II $(2 \times 2)$

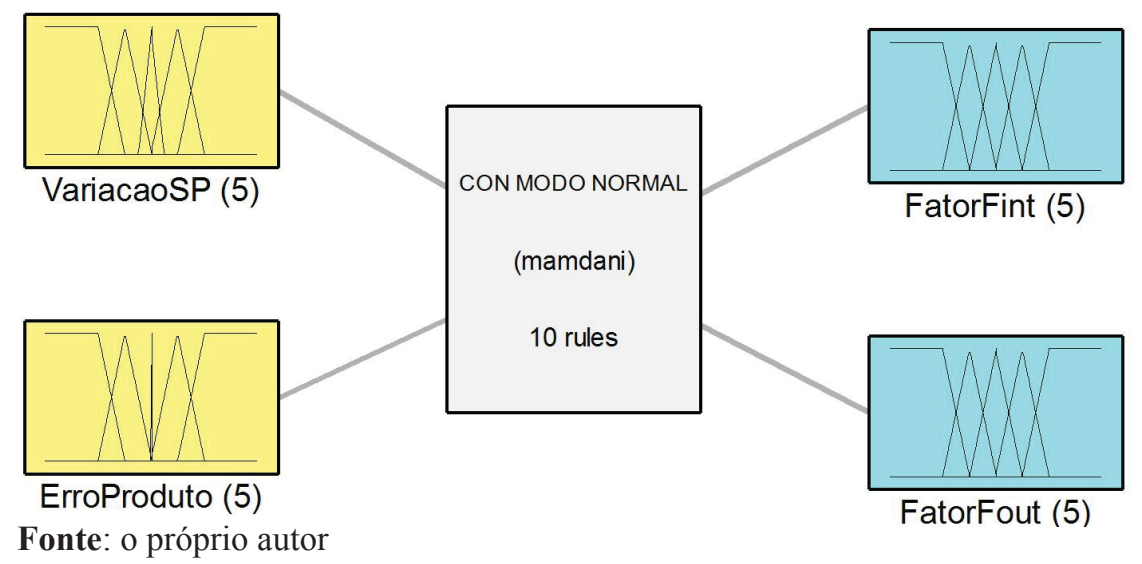

Figura 12 mostra a ponderação das dez regras do controlador II. Este controlador opera apenas em condições normais a ponderação das regras mantevese robusta nas duas campanhas de fermentação. 
Figura 12 - Ponderação das Regras do Controlador 02 da Segunda Arquitetura de Controle Ajustada para as Campanhas 01 e 02

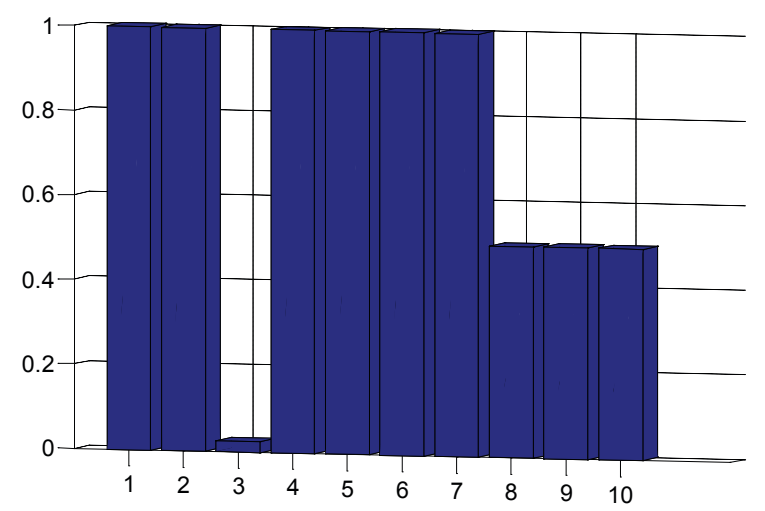

Fonte: o próprio autor

Exemplo de umas das superfícies de controle do controlador II. Figuras 13 e 14.

Figura 13 - Exemplo de superfície de controle, controlador II (Fin)

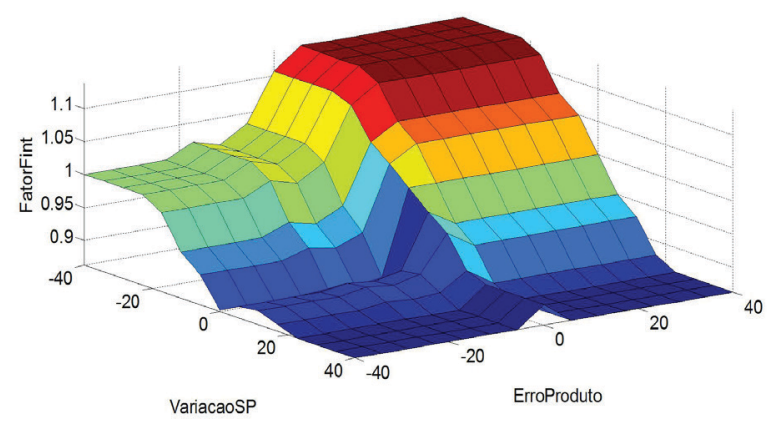

Fonte: o próprio autor

Figura 14: Exemplo de superfície de controle, Fonte: o próprio autor controlador II (Fout)

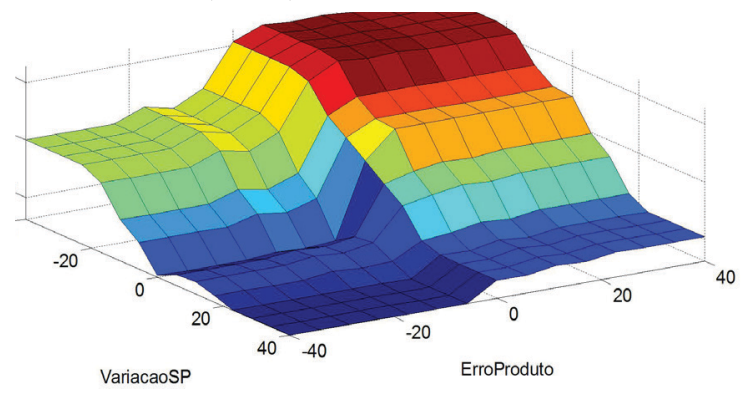

\section{Resultados de simulação}

Para sugerir a robustez do sistema proposto; o mesmo foi aplicado em duas campanhas de fermentação diferentes, e comparadas com o controle clássico PID. Para quantificar os resultados; foram avaliados quatro fatores de desempenho, o erro acumulado das concentrações de produto, substrato, biomassa e a somatória das ultrapassagens dos valores limitados para o volume.

Para simplificação dos termos, os controladores serão chamados de forma resumida FuzzyPID. A comparação dos resultados foi utilizada como referência à somatória do erro (ITAE) na concentração do produto, substrato e biomassa, além da somatória das ultrapassagens dos valores determinados como limite para o volume, essa referência foi denominada como Erro Total.

As tabelas 7, 8, 9 e 10 mostram os resultados obtidos para as campanhas 01 e 02, nas duas arquiteturas de controle, respectivamente para as concentrações do produto, substrato e biomassa, por último o resultado do volume.

Tabela 7 - Resultados de Simulação, para a Concentração do Produto Fermentado

\begin{tabular}{cccc}
\hline $\begin{array}{c}\text { Produto } \\
(\mathrm{g} / \mathbf{l})\end{array}$ & PID & $\begin{array}{c}\text { Arquitetura } \\
\mathbf{0 1}\end{array}$ & $\begin{array}{c}\text { Arquitetura } \\
\mathbf{0 2}\end{array}$ \\
\hline $\begin{array}{c}\text { Erro Total P } \\
\text { Campanha 01 }\end{array}$ & $\mathbf{1 1 1 , 3 9}$ & $\mathbf{9 8 , 5 7}$ & $\mathbf{7 5 , 7 2}$ \\
Erro Total P & $\mathbf{2 3 5 , 1 6}$ & $\mathbf{2 1 8 , 9 4}$ & $\mathbf{2 1 5 , 4 2}$ \\
Campanha 02 & & &
\end{tabular}

Observa-se na tabela 7, que as duas arquiteturas de controle supervisório propostas no trabalho, apresentaram redução no erro acumulado, em relação ao controle PID, e que a arquitetura que apresentou melhor desempenho para variável controlada (Produto), foi à segunda com uma evolução de 32\% na campanha 01 e $8,4 \%$ na campanha 02 .

Fonte: o próprio autor 
Tabela 8 - Resultados de Simulação, para a Concentração do Substrato

\begin{tabular}{rccc}
\hline $\begin{array}{c}\text { Substrato } \\
(\mathbf{g} / \mathbf{l})\end{array}$ & PID & $\begin{array}{c}\text { Arquitetura } \\
\mathbf{0 1}\end{array}$ & $\begin{array}{c}\text { Arquitetura } \\
\mathbf{0 2}\end{array}$ \\
\hline Erro Total S & $\mathbf{1 0 , 7 9}$ & $\mathbf{0 , 2 9}$ & $\mathbf{0 , 2 8}$ \\
Campanha 01 & & & \\
Erro Total S & $\mathbf{0 , 4 1}$ & $\mathbf{0}$ & $\mathbf{0}$ \\
Campanha 02 & & &
\end{tabular}

Fonte: o próprio autor

Na tabela 8, observa-se que, na campanha 01 as arquiteturas 1 e 2 apresentaram, mesmo que simulados, um bom desempenho para a função de proteção do Substrato. Já na campanha 02 as duas arquiteturas conseguiram anular o erro na proteção do Substrato. Na tabela 9 observa-se que a variável Biomassa não apresentou problemas para o controle do processo em nenhumas das arquiteturas.

Tabela 9 - Resultados de Simulação, para a Concentração de Biomassa

\begin{tabular}{cccc}
\hline $\begin{array}{c}\text { Biomassa } \\
(\mathbf{g} / \mathbf{l})\end{array}$ & PID & $\begin{array}{c}\text { Arquitetura } \\
\mathbf{0 1}\end{array}$ & $\begin{array}{c}\text { Arquitetura } \\
\mathbf{0 2}\end{array}$ \\
\hline $\begin{array}{c}\text { Erro TotalC } \\
\text { Campanha 01 }\end{array}$ & $\mathbf{0}$ & $\mathbf{0}$ & $\mathbf{0}$ \\
Erro TotalC & $\mathbf{0}$ & $\mathbf{0}$ & $\mathbf{0}$ \\
Campanha 02 & & &
\end{tabular}

Fonte: o próprio autor

A tabela 10 mostra que, a arquitetura 01 teve o melhor resultado para a proteção do volume do tanque, conseguindo anular o erro na campanha 01 e reduzindo $70 \%$ na campanha 02 .

Tabela 10 Resultados de Simulação para o volume do Tanque

\begin{tabular}{cccc}
\hline $\begin{array}{c}\text { Produto } \\
(\mathbf{g} / \mathbf{l})\end{array}$ & PID & $\begin{array}{c}\text { Arquitetura } \\
\mathbf{0 1}\end{array}$ & $\begin{array}{c}\text { Arquitetura } \\
\mathbf{0 2}\end{array}$ \\
\hline $\begin{array}{c}\text { Erro TotalV } \\
\text { Campanha 01 }\end{array}$ & $\mathbf{8 9 , 0 1}$ & $\mathbf{0}$ & $\mathbf{0 , 0 6}$ \\
Erro TotalV & $\mathbf{1 3 , 9 6}$ & $\mathbf{4 , 1 0}$ & $\mathbf{4 , 2 5}$ \\
Campanha 02 & & & \\
\hline
\end{tabular}

Nota-se que o erro acumulado foi melhor com a utilização dos controles Fuzzy-PID.

Observa-se a dificuldade de ajuste nas duas arquiteturas citadas, mesmo que o desenvolvimento foi de forma gradativa, o ajuste das funções pertinência, das regras e dos seus pesos foram feitos observando o comportamento dinâmico do sistema. E; verificando desempenho, um processo repetitivo e exaustivo, considerando as dificuldades inerentes desse tipo de processo. As figuras 15, 16, 17 e mostram o comportamento dinâmico de todas as variáveis dos processos.

Figura 15 - Resposta dinâmica controladores PID (campanha 1)

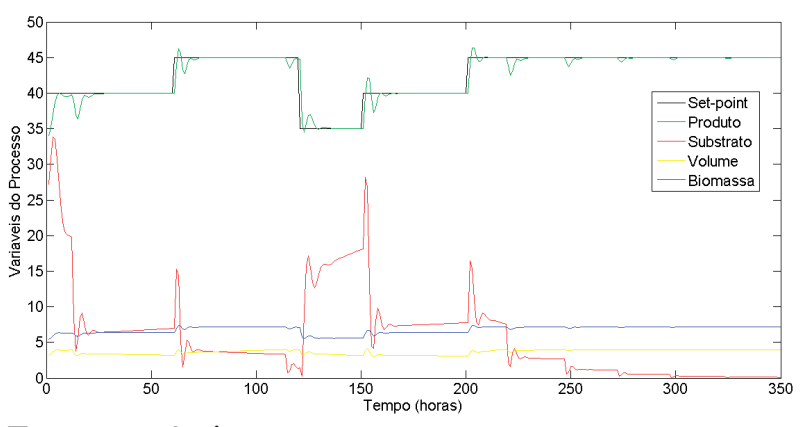

Fonte: o próprio autor

Figura 16 - Resposta dinâmica controladores FUZZY-PID I (campanha 1)

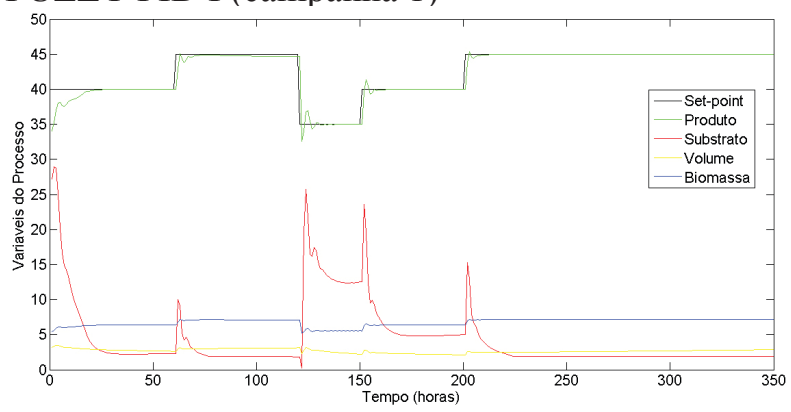

Fonte: o próprio autor

Fonte: o próprio autor 
Figura 17 - Resposta dinâmica controladores FUZZY-PID II (campanha 1)

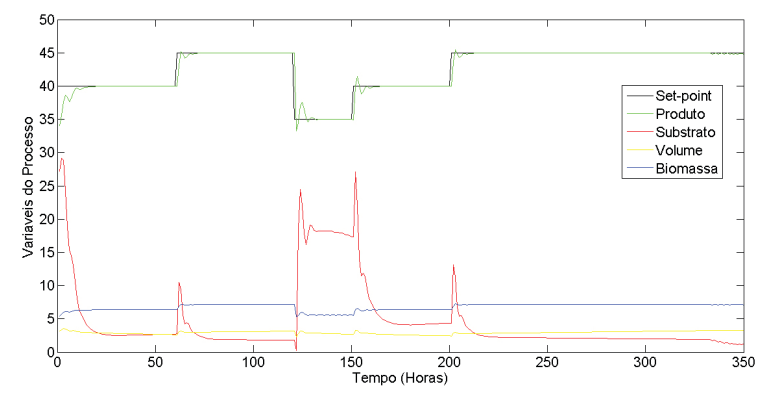

Fonte: o próprio autor

A principal variável controlada (Produto) praticamente sobrepõe os valores de set-points das campanhas. As figuras 18, 19, 20 e mostram as dinâmicas dos quatro controladores na campanha 2.

Figura 18 - Resposta dinâmica controladores PID (campanha

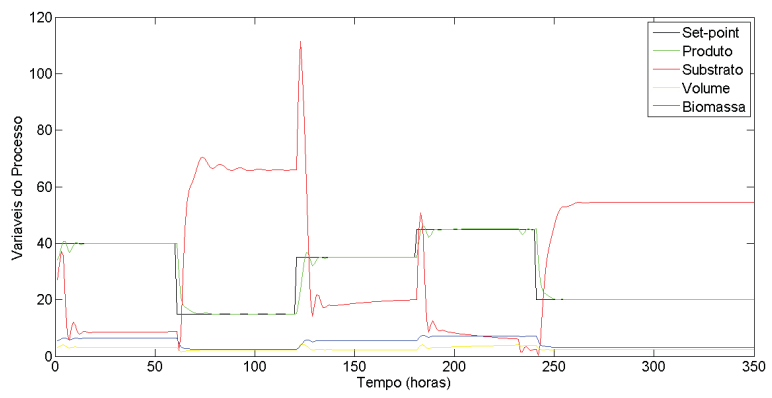

Fonte: o próprio autor

A tabela 10 mostra que, a arquitetura 01 teve o melhor resultado para a proteção do volume do tanque, conseguindo anular o erro na campanha 01 e reduzindo $70 \%$ na campanha 02 .

Figura 19 - Resposta dinâmica controladores FUZZY-PID I (campanha 2)

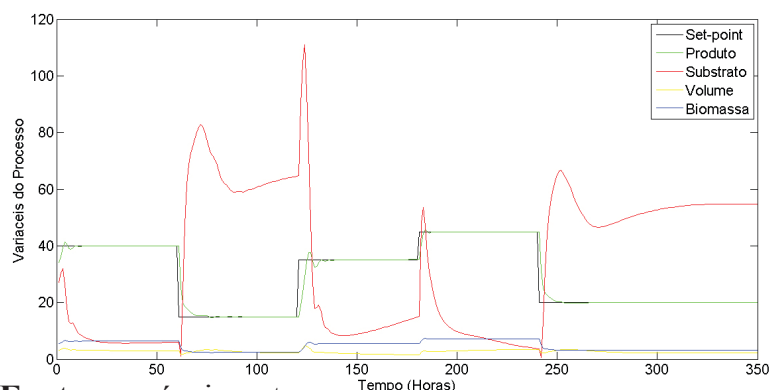

Fonte: o próprio autor
Figura 20 - Resposta dinâmica controladores FUZZY-PID II (campanha 2)

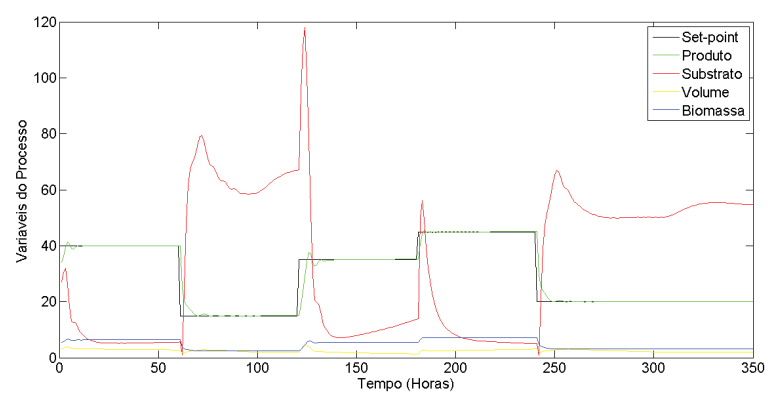

Fonte: o próprio autor

\section{Conclusões}

Esse trabalho apresentou arquiteturas de controladores, com funções de um sistema supervisório baseado em lógica Fuzzy Ponderada e controle clássico (PID) com duas propostas distintas de arquiteturas, aplicada a um processo de fermentação alcoólica multivariável, o qual tem alto nível de complexidade e consequentemente de controle porque apresenta não linearidade, fase não mínima e tempo de acomodação.

Como foi apresentado nos resultados simulados, através das tabelas com os valores numéricos e das figuras, com comportamento dinâmico do processo, as três arquiteturas de controle supervisório apresentaram um resultado satisfatório em relação ao controle clássico PID.

Outra vantagem do sistema proposto é a possibilidade de disparar alarmes para condições adversas de operação, como realizado na arquitetura 02, podendo trocar de controlador Fuzzy, resultando em ganhos consideráveis para o sistema de fermentação alcoólica. Desse modo, podemos sugerir que o sistema supervisório, ainda que inicial, e controlador Fuzzy pode ser viável para esse tipo de processo entre outros processos multivariáveis e não lineares.

É necessário ressaltar que, para trabalhos futuros poderão ser acrescentadas novas funcionalidades no sistema supervisório e compará-lo com outras arquiteturas de controle, como: Redes Cognitivas Dinâmicas (DCNs) e Sistema de Inferência NeuroFuzzy Adaptativo (ANFIS). Também poderá ser 
construída uma Interface Homem Máquina (IHM). E tornando-se uma arquitetura de controle eficiente e consistente, a qual servirá como base teórica para aplicações em outros processos.

Fazer uma inspeção quantitativa no nível de correlação (observável nas equações diferenciais do processo) das variáveis.

E, finalmente pode-se implementar as arquiteturas de controle propostas, em especial a 2, para se avaliar desempenho em um processo de fermentação real.

Espera-se ter contribuído, ainda que com resultados simulados, com uma proposta de sistema supervisório Fuzzy-PID para processos industriais.

\section{Referências}

ALISSON M. S. Sistemas Neuro-Fuzzy Evolutivos: Novos Algoritmos de Aprendizado e Aplicações. 2014. Tese (Doutorado) - Universidade Federal de Minas Gerais. Escola de Engenharia. Programa de Pós-Graduação em Engenharia Elétrica.

ARRIAGA-DE-VALLE, E.; DIECK-ASSAD, G. Modeling and Simulation of a Fuzzy Supervisory Controller for an Industrial Boiler. 2006. Disponivel em: $<$ http://sim.sagepub. com/content /82/12/841.short>. Acesso em: 3 nov. 2014.

FABRO, J. A. Uma abordagem neuro-nebulosa de controle preditivo aplicada a processos multi-estágios. 2003. Tese (Doutorado em Engenharia Elétrica e Informática Industrial) - Centro Federal de Educação Tecnológica do Paraná, Curitiba.

FERNANDES, R. T. Supervisão de um sistema híbrido eólico/diesel usando lógica Fuzzy. 2005. Dissertação (Mestrado em Engenharia Elétrica) - Universidade Federal do Mato Grosso do Sul, Campo Grande.

GOMIDE, F. A. C.; GUDWIN, R. R. Modelagem, Controle, Sistemas e Lógica Fuzzy. SBA Controle \& Automação, Campinas, v. 4, n. 3, p. 97-115, set/out. 1994.

GOMIDE, F. A. C.; PEDRYCZ, W. An introduction to fuzzy sets: analysis and design. 2. ed. Massachusetts: Bradford, 2006.
KASABOV, N. K. Foundations of neural networks, fuzzy systems, and knowledge engineering. Massachusetts London, England: The MIT Press Cambridge, 1998.

MAHER, M. Modélisation elaboration estimation et de commander: application à um bioprocédé. 1995. Tese Université Paul Sabatier, LAAS/CNRS, Toulouse.

MENDONÇA, M. Uma contribuição ao desenvolvimento de sistemas inteligentes utilizando redes cognitivas dinâmicas. 2011. Tese (Doutorado em Engenharia Elétrica e Informática Industrial) - Universidade Tecnológica Federal do Paraná, Curitiba.

PASSINO, M. K.; YOURKOVICH, S. Fuzzy control. Menlo Park: Addison-Wesley, 1997.

SHAW, I. S.; SIMÕES, M. G. Controle e modelagem fuzzy. 2. ed. São Paulo: Edgard Blucher LTDA, 2004.

WANG, R.; LOU, F.; HUANG, X.; LI, P. New elevator energy feedback control system design based on fuzzy PID controller. Power Engineering and Automation Conference (PEAM), IEEE, Sept. 2012.

YAMAKAWA, E. K. Sistema de controle nebuloso para bancos de capacitores automáticos aplicados em alimentadores de distribuição de energia elétrica. 2007. Dissertação (Mestrado em Engenharia Elétrica) Universidade Federal do Paraná, Curitiba.

YESIL, E.; DODURKA, F.; SAKALLI, A.; GUZAY, C., OZTURK, C. Self-Tuning PIController via Fuzzy Cognitive Maps. 2013. (AIAI) Artificial Intelligence Applications and Innovations, Springer, Ifip, September. 2013.

YANG L.; CAI G.; CHENG T.; YANG G., Design and simulation for ball mill load control based on weighted fuzzy control algorithm, Computer Application and System Modeling (ICCASM), 2010 International Conference on , vol.14, no., pp.V14-458,V14-461, 22-24 Oct. 2010.

ZADEH, L. A. An introduction to fuzzy logic applications in intelligent systems. Boston: Kluwer Academic Publisher, 1992.

Recebido em 16 Fevereiro, 2015 - Received on February 16, 2015 Aceito em 19 Agostoo, 2015 - Accepted on August 19, 2015 